\title{
Avaliação de Interfaces por Usuários Finais: Mensurando a Qualidade Afetiva e o Impacto na Aprendizagem de Conteúdos
}

\author{
Rozelma Soares de França \\ Universidade de Pernambuco - Garanhuns, PE - Brasil \\ Centro de Informática - Universidade Federal de Pernambuco - Recife, PE - Brasil \\ E-mail: rozelma.soares@gmail.com \\ Paulo Ricardo Bezerra da Silva Universidade de \\ Pernambuco - Garanhuns, PE - Brasil E-mail: \\ pauloricardo.pr00@gmail.com \\ Haroldo José Costa do Amaral Universidade de \\ Pernambuco - Garanhuns, PE - Brasil E-mail: \\ haroldo.amaral@gmail.com \\ Mirtes Ribeiro de Lira \\ Universidade de Pernambuco - Nazaré da Mata, PE - Brasil \\ E-mail:mirtesrl@uol.com.br
}

Resumo: Este artigo propõe um método para avaliação de interfaces em softwares educativos, envolvendo estudantes, em ambiente escolar. A proposta é baseada no SAM, um instrumento de avaliação iconográfico que contempla questões relacionadas à qualidade afetiva de sistemas computacionais, e na taxonomia de objetivos educacionais de Bloom. Experimentos realizados para a avaliação de interfaces de um software educativo voltado ao ensino da Lógica de Programação demonstram a viabilidade da proposta, permitindo identificar a satisfação dos usuários, a cada interface projetada, e o seu impacto na aprendizagem dos conteúdos contemplados.

Palavras-chave: Avaliação de interfaces; Qualidade afetiva de interfaces; Taxonomia de Bloom; Aprendizagem de conteúdos.

\section{Evaluation of Interfaces for End Users: Measuring the Affective Quality and the Impact Learning Content}

Abstract: This paper proposes a method for evaluating educational software interfaces, involving students in school environment. The proposal is based on SAM, an assessment instrument that includes iconographic issues related to quality of affective computing systems, and the Bloom's taxonomy of educational objectives. Experiments were carried out to evaluate educational software interfaces applied to teaching Logic Programming and demonstrate the feasibility of the proposal, allowing identifying user satisfaction, each designed interface, and its impact on learning of content covered.

Keywords: Assessment interfaces; Quality affective interfaces; Bloom's Taxonomy; Learning content. 


\section{Introdução}

O design de software educativo traz novos requisitos para o processo de desenvolvimento e avaliação de recursos didáticos. Nesse sentido, um dos aspectos fundamentais para o seu sucesso reside na qualidade das interfaces com os usuários (PRATES et al., 2003). As interfaces influenciam na maneira como o usuário percebe e apropria-se do conteúdo, contribuindo para um bom desempenho nas atividades, ou, contrariamente, impedindo ou inviabilizando os processos cognitivos (PASSOS, 2011).

O ciclo de desenvolvimento de software envolve várias etapas, incluindo a avaliação de interfaces (SOARES \& BARANAUSKAS, 2005), cujos objetivos são: i) avaliar as funcionalidades do software; ii) avaliar o efeito das interfaces junto ao usuário e iii) identificar problemas específicos no software (ROCHA \& BARANAUSKAS, 2003). Tais informações são importantes para que o designer possa melhorar e adequar o design do software às necessidades dos usuários. No que concerne ao design de softwares educativos, também, deve ser considerado o impacto do uso de uma interface, na aprendizagem de conteúdos específicos (GOMES, 2005).

Na literatura, há relatos de adaptação de métodos de usabilidade para uso com crianças (HANNA et al., 1997; VAN KESTEREN et al., 2003; DONKER et al, 2004). Em aplicações educacionais, problemas de usabilidade podem criar barreiras que dificultam que o usuário atinja os objetivos de aprendizagem pretendidos alcançar com a utilização do software. No entanto, além de avaliar este aspecto, faz-se necessário explorar a eficácia pedagógica do sistema em uso. Assim, neste artigo, será proposto um método para avaliação de interfaces em softwares educativos que possam ser utilizados para apoiar o processo de ensino e aprendizagem de conteúdos de dada disciplina.

O método procura avaliar: i) a qualidade afetiva de suas interfaces e ii) o impacto do uso das interfaces na aprendizagem de conteúdos. O primeiro item é justificado pelas pesquisas nas áreas de Interação Humano-Computador e de Sistemas de Informação que sugerem que a percepção da afetividade de uma interface tem impacto positivo na forma como usuários percebem a usabilidade de um sistema (SCHENKMAN et al., 2000). O segundo está respaldado na importância em avaliar a aprendizagem provida por softwares educativos (GOMES, 2005).

Um importante requisito considerado no método proposto é a sua fácil aplicação e interpretação, por parte dos usuários. Por ser baseado em um instrumento de avaliação iconográfico, que não demanda leitura, a proposta mostra-se adequada ao perfil de usuários, alunos da educação básica. Outro requisito é que o método possibilita identificar, na percepção do usuário, o nível de conhecimento dos estudantes, sobre um conteúdo, antes e depois do uso do software educativo, permitindo mensurar o impacto da utilização de suas interfaces na aprendizagem dos conteúdos trabalhados. Este último requisito está ancorado na Taxonomia de Bloom, uma taxonomia de objetivos educacionais proposta por Bloom et al. (1956).

O restante do artigo está organizado como segue: a Seção 2 apresenta o método proposto e suas bases teóricas. A Seção 3 descreve a aplicação do método na avaliação das interfaces de um software para apoio ao ensino da Lógica de Programação e os seus resultados. Por fim, na Seção 4, são feitas algumas considerações acerca deste trabalho.

\section{Um método para avaliação de interfaces educativas com usuários finais}

A qualidade da interface é fundamental para que sistemas interativos possam ser utilizados com sucesso, de forma que a sua avaliação é necessária. Há dois tipos de 
avaliação (PREECE et al., 2005): i) Avaliações formativas que são realizadas durante o design, visando verificar se o produto continua a atender às necessidades dos usuários e ii) Avaliações somativas realizadas para avaliar o sucesso de um produto finalizado.

O método de avaliação de interfaces proposto pode ser utilizado na análise de projetos já finalizados. Ele foi idealizado levando em conta o uso do SAM (Self Assessment Manikin) (LANG, 1980) e um questionário com perguntas baseadas em níveis da taxonomia de Bloom, como ilustra a Figura 1.

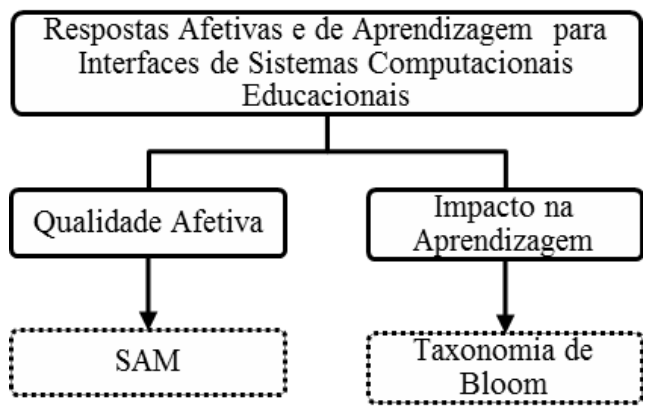

Figura 1 - Bases para Avaliação de Interfaces Educativas com Usuários Finais

\subsection{Avaliação da qualidade afetiva}

Como instrumento de medida para avaliação da qualidade afetiva de interfaces de softwares educativos, propõe-se o uso do SAM (Self Assessment Manikin), um método de avaliação, não verbal, desenvolvido por Lang (1980), que contempla questões relacionadas à qualidade afetiva de um sistema computacional. Por meio dele, é possível identificar a motivação e a satisfação em seu uso.

Para aplicá-lo, um questionário não verbal foi criado, devendo ser preenchido para cada interface que se deseja avaliar, sendo dividido em três partes, como ilustra a Figura 2: Satisfação, Motivação e Sentimento de Controle. Em cada parte, há nove ícones (lado direito da Figura 2), onde: o quinto representa uma resposta neutra do participante; os quatro primeiros representam alguns sentimentos existentes no lado esquerdo do quadro localizado no lado esquerdo da Figura 2 (por exemplo, para a parte Motivação, sentimentos, tais como: Calmo, Relaxado, Vagaroso, Lento, Sono e Tranquilo); os quatro últimos ícones representam os sentimentos existentes no lado direito do mesmo quadro (por exemplo, para a parte Motivação, sentimentos, tais como: Animado, Estimulado, Frenético, Nervoso e Agitado). O mesmo raciocínio é usado para as partes Satisfação e Sentimento de Controle.

\begin{tabular}{|l|l|l|l|l|l|}
\hline \multicolumn{3}{|c|}{ Satisfação } & & \\
\hline $\begin{array}{l}\text { Infeliz } \\
\text { Nervoso } \\
\text { Irritado } \\
\text { Insatisfeito } \\
\text { Melancólico } \\
\text { Desesperado } \\
\text { Entediado }\end{array}$ & $\begin{array}{l}\text { Feliz } \\
\text { Sorridente } \\
\text { Prazer } \\
\text { Satisfeito } \\
\text { Contente } \\
\text { Otimista } \\
\text { Esperançoso }\end{array}$ & &
\end{tabular}

Figura 2 - Questionário SAM 


\subsection{Avaliação do impacto das interfaces na aprendizagem de conteúdos}

No método proposto, considerou-se os princípios da Taxonomia de Bloom (BLOOM et al., 1956; ANDERSON \& KRATHWOHL, 2001), para avaliar o impacto do uso das interfaces na aprendizagem dos estudantes, constituída por três domínios: Cognitivo, Afetivo, Psicomotor. Entretanto, o domínio Cognitivo é o mais conhecido e utilizado, onde diversos educadores apóiam-se nos pressupostos teóricos desse domínio para definirem, em seus planejamentos educacionais, objetivos, estratégias e sistemas de avaliação (FERRAZ et al., 2010).

No domínio Cognitivo, os objetivos foram agrupados em seis diferentes categorias e são apresentados numa hierarquia de complexidade e dependência, do mais simples ao mais complexo. Assim, para ascender a uma nova categoria, é preciso ter obtido um adequado desempenho na anterior, pois cada uma utiliza capacidades adquiridas nos níveis anteriores. As categorias desse domínio são: Conhecimento; Compreensão; Aplicação; Análise; Síntese; e Avaliação.

Com a incorporação de novos conceitos, recursos e teorias ao campo educacional, bem como os avanços psico-pedagógicos e tecnológicos ocorridos e a publicação de diversas experiências de sucesso no uso efetivo da taxonomia, observouse a necessidade da reavaliação e releitura dos pressupostos teóricos que sustentam a pesquisa original, apresentada em 1956 (BLOOM et al., 1956). Dessa forma, foram realizadas a revisão e a atualização da Taxonomia de Bloom (ANDERSON \& KRATHWOHL, 2001).

$\mathrm{Na}$ taxonomia revisada, a base das categorias foi mantida, permanecendo seis categorias, entretanto, ao separar, conceitualmente, o conhecimento do processo cognitivo, ocorreram às seguintes mudanças (KRATHWOHL, 2002): i) os aspectos verbais utilizados na categoria Conhecimento foram mantidos, mas esta foi renomeada para Lembrar; ii) Compreensão foi renomeada para Entender; iii) Aplicação, Análise e Avaliação, foram alteradas para a forma verbal Aplicar, Analisar e Avaliar, respectivamente, por expressarem melhor a ação pretendida e serem condizentes com o que se espera de resultado a determinado estímulo de instrução; iv) Síntese trocou de lugar com Avaliação (Avaliar) e foi rebatizada para a forma verbal Criar; v) os nomes das subcategorias existentes foram alterados para verbos no gerúndio (Quadro 1).

Quadro 1 - Estrutura do processo cognitivo na Taxonomia de Bloom revisada

\section{Lembrar}

Relacionado a reconhecer e reproduzir ideias e conteúdos. Reconhecer requer distinguir e selecionar uma determinada informação e reproduzir ou recordar está mais relacionado à busca por uma informação relevante memorizada. Representado pelos seguintes verbos no gerúndio: Reconhecendo e Reproduzindo. Entender

Relacionado a estabelecer uma conexão entre o novo e o conhecimento previamente adquirido. A informação é entendida quando o aprendiz consegue reproduzi-la com suas "próprias palavras". Representado pelos seguintes verbos no gerúndio: Interpretando, Exemplificando, Classificando, Resumindo, Inferindo, Comparando e Explicando.

\section{Aplicar}

Relacionado a executar ou usar um procedimento numa situação específica e pode também abordar a aplicação de um conhecimento numa situação nova. Representado pelos seguintes verbos no gerúndio: Executando e Implementando.

\section{Analisar}

Relacionado a dividir a informação em partes relevantes e irrelevantes, importantes e menos importantes e entender a inter-relação existente entre as partes. Representado pelos seguintes verbos no gerúndio: Diferenciando, Organizando, Atribuindo e Concluindo.

\section{Avaliar}

Relacionado a realizar julgamentos baseados em critérios e padrões qualitativos e quantitativos ou de eficiência e eficácia. Representado pelos seguintes verbos no gerúndio: Checando e Criticando. 


\section{Criar}

Significa colocar elementos junto com o objetivo de criar uma nova visão, uma nova solução, estrutura ou modelo utilizando conhecimentos e habilidades previamente adquiridos. Envolve o desenvolvimento de ideias novas e originais, produtos e métodos por meio da percepção da interdisciplinaridade e da interdependência de conceitos. Representado pelos seguintes verbos no gerúndio: Generalizando, Planejando e Produzindo.

$$
\text { Fonte: Ferraz et al. (2010) }
$$

No método de avaliação aqui proposto, os três primeiros níveis da Taxonomia de Bloom revisada são levados em consideração: Lembrar, Entender e Aplicar. O questionário para avaliação do impacto do uso das interfaces de um sistema na aprendizagem de conteúdos específicos foi inspirado no trabalho de Savi (2011) que utiliza questionário para avaliar jogos educativos. Após a interação dos estudantes com as interfaces do sistema educativo, é solicitado que cada um deles indique seu nível de conhecimento, numa escala entre 0 e 10 , sobre os conteúdos tratados, tendo em vista os três primeiros níveis da Taxonomia de Bloom, antes e após o uso do software, como mostra o Quadro 2.

Quadro 2 - Questionário para avaliação das interfaces na aprendizagem

Atribua uma nota de 0,0 a 10,0 para seu nível de conhecimento antes e depois da interação com as interfaces do sistema, aos conteúdos listados no quadro abaixo.

\begin{tabular}{|l|c|c|c|c|c|c|}
\hline \multirow{2}{*}{ Conteúdo } & \multicolumn{2}{c|}{ Lembrar } & \multicolumn{2}{c|}{ Entender } & \multicolumn{2}{c|}{ Aplicar } \\
\cline { 2 - 7 } & Antes & Depois & Antes & Depois & Antes & Depois \\
\hline Conteúdo & & & & & & \\
\hline Conteúdo $_{2}$ & & & & & & \\
\hline Conteúdo $_{\mathrm{n}}$ & & & & & & \\
\hline
\end{tabular}

Após a avaliação e análise dos resultados, é possível identificar se as interfaces produziram efeitos positivos na aprendizagem. Para isso, basta comparar as médias entre a percepção do nível de conhecimento do estudante antes e após a interação. As interfaces com conteúdos relacionados a objetivos de aprendizagem que obtiverem maiores médias, após a interação, podem ser classificadas como aquelas que mais contribuíram para a aprendizagem, de acordo com a percepção dos alunos.

Tabela 1 - Medida do nível de conhecimento dos estudantes

\begin{tabular}{|c|c|c|c|c|}
\hline $\begin{array}{l}\text { Nível de conhecimento } \\
\text { sobre os conteúdos }\end{array}$ & Estado & Média & $\begin{array}{l}\text { Desvio } \\
\text { Padrão }\end{array}$ & $\begin{array}{c}\text { Diferença } \\
\text { Depois-Antes }\end{array}$ \\
\hline \multirow{2}{*}{ Lembrar Conteúdo $_{\mathrm{n}}$} & Antes & & & \\
\hline & Depois & & & \\
\hline \multirow{2}{*}{ Entender Conteúdo ${ }_{\mathrm{n}}$} & Antes & & & \\
\hline & Depois & & & \\
\hline \multirow{2}{*}{ Aplicar Conteúdo ${ }_{\mathrm{n}}$} & Antes & & & \\
\hline & Depois & & & \\
\hline
\end{tabular}

\section{Aplicação do método proposto}

Para demonstrar a viabilidade do método proposto, um estudo de caso foi realizado numa escola pública de ensino situada no agreste de Pernambuco. A avaliação deu-se no sistema LOOP, descrito na Subseção 3.1.

\subsection{Sistema LOOP}

O LOOP (FRANÇA \& AMARAL, 2012) é um sistema de apoio ao aprendizado, desenvolvido por alunos do curso de Licenciatura em Computação da Universidade de 
Pernambuco (UPE), para apoiar o ensino da Lógica de Programação a alunos do Ensino Médio. Os autores defendem que o aprendizado desses conceitos, na Educação Básica, auxilia os estudantes no raciocínio, na compreensão de conceitos básicos, além de melhor prepará-los para o entendimento de tópicos mais avançados em computação. No entanto, as metodologias aplicadas ao ensino da Lógica de Programação e até o grau de dificuldade intrínseco da área muitas vezes repercutem negativamente, desmotivando o estudante no processo de aprendizagem.

Com o objetivo de auxiliar os alunos no aprendizado dos fundamentos da Lógica de Programação e possibilitar um melhor entendimento dos conceitos de forma lúdica, o sistema LOOP foi proposto e desenvolvido. Para que se conseguisse atingir os objetivos educacionais e de design desejados, ele foi desenvolvido dentro da abordagem do Design de Interação (PREECE et al., 2005) e do Design Instrucional (FILATRO, 2008).

O sistema é estruturado em seis módulos. Cada módulo é formado por uma subpágina contendo um mapa conceitual relativo ao conteúdo tratado, uma subpágina de material de apoio com indicações de links a outros sites que abordam o conteúdo, e uma subpágina composta por desafios que tratam de conceitos da Lógica de Programação.

Em cada módulo, há um conjunto de desafios, refletindo a complexidade crescente dos conteúdos abordados. A cada desafio, problemas são propostos, no intuito do usuário descobrir uma solução algorítmica, consolidando, assim, a sua aprendizagem relativa aos conteúdos do desafio em questão. Caso o usuário seja bem sucedido no desafio, uma melodia regida por personagens, atrelada e acompanhada de cores, é produzida. Caso contrário, um personagem desafina em parte da melodia assinalando ao usuário que a solução não está correta. Considerando que os desafios, através das suas interfaces, possibilitam a composição de uma melodia, esta é construída gradativamente, a cada módulo, pelos seus personagens, de acordo com a aprendizagem dos conteúdos propostos trabalhados pelo usuário.

Os módulos que compõem o LOOP contemplam os seguintes conteúdos:

- Módulo 1: Variáveis, constantes, tipos de dados, comandos de leitura e escrita;

- Módulo 2: Comandos de atribuição e expressões aritméticas;

- Módulo 3: Expressões booleanas (operadores booleanos, relacionais, de igualdade, considerando a ordem de prioridade e associatividade) e estruturas de decisão;

- Módulo 4: Estruturas de repetição;

- Módulo 5: Arrays;

- Módulo 6: Modularidade: funções e procedimentos, considerando a passagem de parâmetros por valor e por referência.

\subsection{Participação e avaliação dos estudantes}

A aplicação do método proposto foi realizada em ambiente escolar, no laboratório de informática de uma escola pública do Estado de Pernambuco, com a participação de vinte e quatro estudantes do Ensino Médio na faixa etária de 15 a 17 anos.

A atividade teve duração aproximada de duas horas e foi iniciada pela explicação do método de avaliação aos estudantes avaliadores. Em seguida, cada aluno recebeu doze questionários SAM's, um para cada interface a ser avaliada, e um questionário para avaliação do impacto das interfaces na aprendizagem dos conteúdos tratados pelo sistema LOOP. 
Para a avaliação da qualidade afetiva, doze telas foram selecionadas para investigação dos elementos de interface. A cada tela projetada, os participantes marcavam com um ' $X$ ' à figura que melhor representava o seu sentimento imediato em relação à tela. Isso ocorreu em cada dimensão de estado de reação emocional: satisfação, motivação e sentimento de controle. Esta avaliação foi realizada de maneira anônima e individual. Como resultado, cada uma das doze telas foi avaliada por vinte e quatro estudantes que indicavam, por meio das figuras que compunham o questionário SAM, a sua reação de afetividade ao que lhe era apresentado.

Num segundo momento, os estudantes avaliadores acessaram o LOOP e trabalharam individualmente durante o preenchimento do questionário que tratava sobre a aprendizagem dos conteúdos abordados pelo sistema em análise.

Ao término da avaliação, todos os participantes reuniram-se para discutir sobre suas dificuldades durante a avaliação e dar sugestões sobre o design das interfaces. Vale ressaltar que, neste momento, a pretensão não era que os estudantes avaliadores chegassem a um consenso, mas que sugerissem melhorias para ações posteriores pelos desenvolvedores do sistema LOOP. Anotações sobre os comentários dos alunos foram realizadas nesta etapa.

\subsection{Resultado da avaliação da qualidade afetiva do sistema LOOP}

Com o intuito de classificar as telas, evidenciando as de maior e menor aceitabilidade, as folhas de respostas foram recolhidas e somamos os votos recebidos para cada tela analisada. A Figura 3 traz um exemplo com uma das doze telas avaliadas.

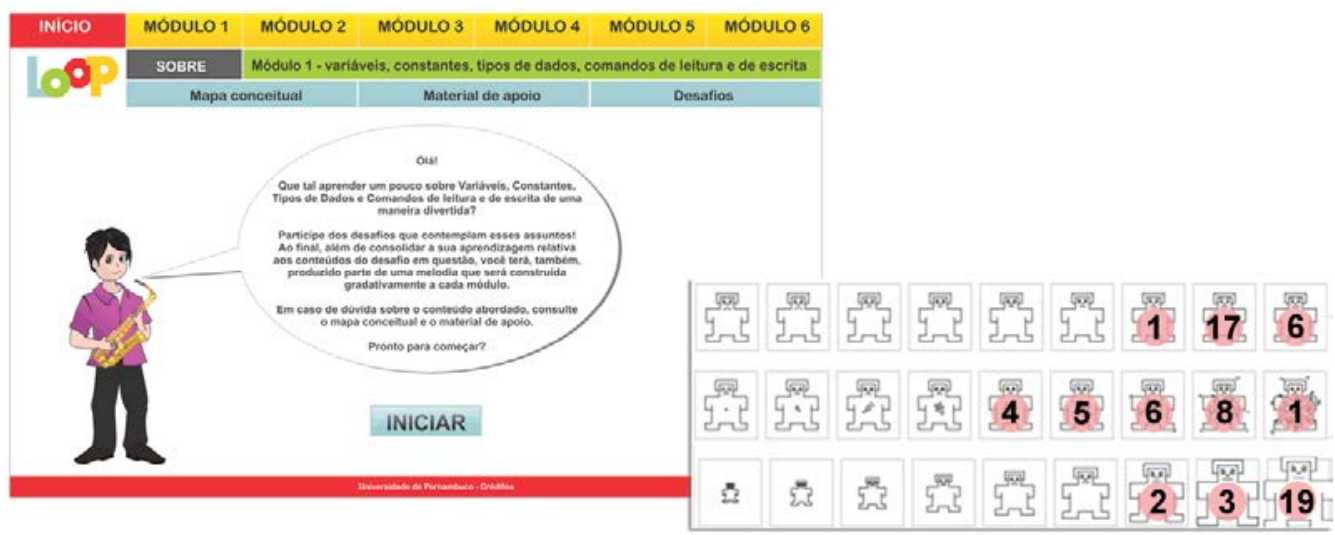

Figura 3 - Interface Inicial do Módulo 1 - Exemplo de respostas compiladas

Para a tela do exemplo, um participante marcou, em seu formulário SAM, a sétima figura da primeira linha (terceira melhor nota na categoria satisfação); dezessete marcaram a oitava figura (segunda melhor nota na categoria satisfação); seis marcaram a nona (melhor nota na categoria satisfação), e assim por diante, de maneira que a soma dos votos de cada linha resultou em vinte e quatro, o número total de participantes. $\mathrm{O}$ mesmo foi feito para as outras telas.

Aos votos, foram atribuídos pontos positivos (V+), negativos (V-) ou neutros (VN). A coluna central recebeu pontuação neutra. Os votos marcados em uma imagem à direita da coluna central receberam pontos positivos, e os assinalados à esquerda receberam pontos negativos.

Todos os pontos foram somados e contabilizados em um resultado geral. Somando-se os pontos obtidos, uma comparação pôde ser feita entre votos positivos, neutros e negativos de cada categoria o que forneceu uma indicação da reação do aluno 
ao objeto analisado, evidenciando a sua qualidade afetiva imediata (HAYASHI et al., 2009). A Tabela 2 mostra a soma dos votos para a Tela Inicial do Módulo 1.

Tabela 2 - Votos da Tela Inicial do Módulo 1

\begin{tabular}{c|c|c|c|c|c|c|c|c}
\hline \multicolumn{3}{c|}{ Satisfação } & \multicolumn{3}{c|}{ Motivação } & \multicolumn{3}{c}{$\begin{array}{c}\text { Sentimento de } \\
\text { Controle }\end{array}$} \\
\hline V- & VN & V+ & V- & VN & V+ & V- & VN & V+ \\
\hline 0 & 0 & 24 & 0 & 4 & 20 & 0 & 0 & 24 \\
\hline
\end{tabular}

\subsection{Resultado da avaliação do impacto das interfaces na aprendizagem dos conteúdos tratados pelo sistema LOOP}

A Tabela 3 apresenta as médias da autoavaliação dos estudantes quanto aos conteúdos tratados no Módulo 1 do LOOP, de acordo com os níveis da Taxonomia de Bloom, antes e depois da interação com as interfaces. A título de exemplificação, serão descritos os resultados da aprendizagem sobre um dos conteúdos contemplados no módulo mencionado: Variáveis.

Tabela 3 - Resultado compilado do nível de conhecimento dos estudantes

\begin{tabular}{l|c|c|c|c}
\hline $\begin{array}{c}\text { Nível de conhecimento } \\
\text { sobre os conteúdos }\end{array}$ & Estado & Média & $\begin{array}{c}\text { Desvio } \\
\text { Padrão }\end{array}$ & $\begin{array}{c}\text { Diferença } \\
\text { Depois-Antes }\end{array}$ \\
\hline \multirow{2}{*}{ Lembrar Variáveis } & Antes & 8,04 & 1,30 & \multirow{2}{*}{1,13} \\
\cline { 2 - 4 } & Depois & 9,17 & 0,92 & \multirow{2}{*}{0,59} \\
\cline { 2 - 4 } Entender Variáveis & Antes & 7,58 & 1,25 & \multirow{2}{*}{0,71} \\
\cline { 2 - 4 } Aplicar Variáveis & Depois & 8,17 & 1,27 & 1,53 \\
\cline { 2 - 4 } & Antes & 7,08 & 1,41 & \\
\cline { 2 - 4 }
\end{tabular}

Como pode ser observado na Tabela 3, os estudantes perceberam um aumento no nível de conhecimento sobre Variáveis em todos os objetivos de aprendizagem do LOOP. As interfaces com maior impacto positivo na aprendizagem foram aquelas que exploraram o conteúdo em questão no nível Lembrar, seguidas pelas que abordaram o conteúdo Variáveis no nível Aplicar e Entender, nesta ordem. Tais resultados evidenciam que as interfaces do sistema LOOP, que abordavam o conteúdo Variáveis, não interferem negativamente na aprendizagem de seus usuários.

\section{Considerações finais}

Este artigo apresentou um método para avaliação de interfaces em softwares educativos, por parte dos usuários finais. Nesse contexto, variáveis de aprendizagem também devem ser consideradas no momento da avaliação do design, já que a maneira como as interfaces de uma aplicação educativa são projetadas podem influenciar na forma como o aluno apropria-se do conteúdo e também interferir em sua aprendizagem.

Para atender aos requisitos de aprendizagem na análise de interfaces, o método proposto buscou aporte teórico em Benjamin Bloom que, juntamente com outros pesquisadores, propuseram, em 1956, a Taxonomia de Bloom, uma taxonomia de objetivos educacionais que pode auxiliar os educadores em tarefas como planejamento e avaliação da aprendizagem. Destaca-se, no entanto, que os resultados do impacto do uso do software na aprendizagem são obtidos tendo em vista a autoavaliação do estudante acerca dos conteúdos abordados, antes e depois da interação com as interfaces. Esses dados são importantes, mas não suficientes para afirmar sobre a qualidade pedagógica e de conteúdos do aplicativo educacional. 
Tendo em vista que compreender o que provoca emoções nos usuários é fundamental para a área de Interação Humano-Computador, pois permite que interfaces sejam projetadas para estimular os estados emocionais desejados, o método de avaliação aqui apresentado contempla também a análise da qualidade afetiva. Na proposta, a avaliação desse tópico foi feita através de um questionário iconográfico que permite mensurar a consciente experiência de emoção do usuário ao interagir com um software. No entanto, para a realização de uma avaliação mais detalhada, outros instrumentos são recomendados, a exemplo de webcam a fim de registrar as expressões faciais do usuário enquanto interage com o software.

Para validar a proposta apresentada, uma avaliação, envolvendo estudantes do Ensino Médio, foi realizada em ambiente escolar. Os alunos envolvidos tiveram participação ativa na avaliação, julgando sobre a qualidade afetiva e o impacto que as interfaces do LOOP têm sobre a aprendizagem de conteúdos da Lógica de Programação. Os resultados demonstraram que as interfaces do sistema analisado propiciam uma experiência de uso agradável aos seus usuários. Além disso, o design projetado favorece a aprendizagem de conteúdos específicos da área de Computação.

\section{Agradecimentos}

Projeto financiado pelo Conselho Nacional de Desenvolvimento Científico e Tecnológico (CNPq).

\section{Referências bibliográficas}

ANDERSON, L. W.; KRATHWOHL, D. R. (Ed.). A taxonomy for learning, teaching, and assessing: A revision of Bloom's taxonomy of educational objectives. New York: Addison Wesley Longman. 2001.

BLOOM, B. S.; ENGELHART, M. D.; FURST, E. J.; HILL, W. H.; KRATHWOHL, D. R.. Taxonomy of educational objectives: The classification of educational goals. Handbook 1: Cognitive domain. New York: David McKay. 1956.

DONKER, A., REITSMA, P. . Usability testing with young children. In: CONFERENCE ON INTERACTION DESIGN AND CHILDREN, College Park, MD, p. 43-48, 2004.

FERRAZ, A. P. DO C. M.; BELHOT, R. V.. Taxonomia de Bloom: revisão teórica e apresentação das adequações do instrumento para definição de objetivos instrucionais. Gest. Prod., São Carlos, v. 17, n. 2, p. 421-431, 2010.

FILATRO, A. Design Instrucional na prática. São Paulo: Pearson Education do Brasil. 2008.

FRANÇA, R. S. ; AMARAL, H. J. C. Proposta de um Jogo Eletrônico Educativo Aplicado ao Ensino da Lógica de Programação. In: CONFERÊNCIA LATINOAMERICANA DE OBJETOS E TECNOLOGIAS DE APRENDIZAGEM, 7, 2012, Guayaquil. Anais. Guayaquil: Comunidade Latinoamericana de Objetos de Aprendizagem, 2012.

GOMES, A. S. Avaliação da aprendizagem com software educativo no projeto interativo. In: CONGRESSO INTERNACIONAL DE ERGONOMIA E USABILIDADE DE INTERFACE HUMANO MÁQUINA, 5, 2005, Rio de Janeiro. Anais. Rio de Janeiro: Pontifícia Universidade Católica do Rio de Janeiro, 2005. 
HANNA, L., RISDEN, K., ALEXANDER, K. . Guidelines for usability testing with children. Interactions, Setembro/Outubro, p. 9-14, 1997.

HAYASHI, E. C. S. ; NERIS, V. P. A. ; MARTINS, M. C. ; BARANAUSKAS, M. C. C. ; PICCOLO, L. S. G. ; COSTA, R. . Avaliando a Qualidade Afetiva de Sistemas Computacionais Interativos no Cenário Brasileiro. In: MELO, A. M.; PICCOLO, L. S. G. ; AVILA, I. M. A. ; TAMBASCIA, C. A.. (Org.). Usabilidade, Acessibilidade e Inteligibilidade Aplicadas em Interfaces para Analfabetos, Idosos e Pessoas com Deficiência - Resultados do Workshop. Usabilidade, Acessibilidade e Inteligibilidade Aplicadas em Interfaces para Analfabetos, Idosos e Pessoas com Deficiência Resultados do Workshop. Campinas: Fundação CPqD. 2009. 55-62p.

KRATHWOHL, D. R. A revision of Bloom's taxonomy: an overview. Theory in Practice, v. 41, n. 4. 2002. 212-218p.

LANG, P. J. . Behavioral treatment and bio-behavioral assessment: computer applications. In: J. B. SIDOWSKI, J. H. JOHNSON; T. A. WILLIAMS (Eds.), Technology in mental health care delivery systems. Norwood, NJ: Ablex, 1980. 119$137 p$.

PASSOS, P. C. S. J. Interad: uma metodologia para design de interface de materiais educacionais digitais. Porto Alegre: Universidade Federal do Rio Grande do Sul, Faculdade de Educação, 2011, 182p. Dissertação de Mestrado.

PRATES, R. O. ; FIGUEIREDO, R. M. V. de; BACH, C. F. Um Modelo de Apoio ao Projeto de Design de Interfaces de Ambientes de Aprendizado. In: WORKSHOP DE INFORMÁTICA NA ESCOLA, 9, 2003, Campinas. Anais. Campinas: Sociedade Brasileira de Computação, 2003.

PRATES, R. O. ; BARBOSA, S. D. J. Avaliação de Interfaces de Usuário - Conceitos e Métodos. In: Coello, J. M. A.; Fabbri, S. C. P. F. (Org.). Jornada de Atualização em Informática do Congresso da Sociedade Brasileira de Computação. Campinas: SBC, 2003, v. 2. 2003. 245-293p.

PREECE, J.; ROGERS, Y.; SHARP, H. Design de interação: Além da interação homem-computador. Porto Alegre: Bookman. 2005.

ROCHA, H. V.; BARANAUSKAS, M. C. C. Design e Avaliação de Interfaces Humano-Computador, ISBN-85-88833-04-2. 2003.161 - 213p.

SAVI, R. Avaliação de jogos voltados para a disseminação do conhecimento. Florianópolis: Universidade Federal de Santa Catarina, Centro Tecnológico, 2011, 238p. Tese de Doutorado.

SOARES, S. C. DE M.; BARANAUSKAS, M. C. C. Avaliação de Interfaces pelo Usuário Final: Alunos em Ambiente Escolar. In: WORKSHOP DE INFORMÁTICA NA ESCOLA, 11, 2005, São Leopoldo. Anais. São Leopoldo: Sociedade Brasileira de Computação, 2005.

SCHENKMAN, B. N.; JONSSON, F.U. Aesthetics and preferences of Web pages. Behaviour \& Information Technology, v. 19, 5, p. 367-377, 2000.

VAN KESTEREN, I. E., BEKKER, M. M., VERMEEREN, A. P. O. S., LLOYD, P. A.. Assessing usability evaluation methods on their effectiveness to elicit verbal comment from children subjects. In: CONFERENCE ON INTERACTION DESIGN AND CHILDREN, Preston, UK, p. 41-49, 2003. 